\title{
The evolution of high-redshift massive black holes
}

\author{
Marta Volonteri ${ }^{1}$, Melanie Habouzit ${ }^{1}$, Fabio Pacucci ${ }^{2}$ \\ and Michael Tremmel ${ }^{3}$ \\ ${ }^{1}$ Institut dAstrophysique de Paris, Sorbonne Universitès, UPMC Univ Paris 6 et CNRS, UMR \\ 7095, 98 bis bd Arago, 75014 Paris, France \\ email: martav@iap.fr, habouzit@iap.fr \\ ${ }^{2}$ Scuola Normale Superiore, Piazza dei Cavalieri, 756126 Pisa, Italy \\ email: fabio.pacucci@sns.it \\ ${ }^{3}$ Department of Astronomy, University of Washington, Seattle, WA \\ email: mjt29@astro.washington.edu
}

\begin{abstract}
Massive black holes (MBHs) are nowadays recognized as integral parts of galaxy evolution. Both the approximate proportionality between MBH and galaxy mass, and the expected importance of feedback from active $\mathrm{MBHs}$ in regulating star formation in their host galaxies point to a strong interplay between MBHs and galaxies. MBHs must form in the first galaxies and be fed by gas in these galaxies, with continuous or intermittent inflows that, at times, can be larger than the Eddington rate. Feedback from supernovae and from the MBHs themselves modulates the growth of the first MBHs. While current observational data only probe the most massive and luminous MBHs, the tip of the iceberg, we will soon be able to test theoretical models of MBH evolution on more "normal" MBHs: the MBHs that are indeed relevant in building the population that we observe in local galaxies, including our own Milky Way.
\end{abstract}

Keywords. quasars: general, black hole physics, galaxies: evolution

\section{Introduction}

Quasars have now been detected up to $z=7$ (Mortlock et al. 2011). The current sample, based on optical and near-infrared data, is characterized by high luminosity and large estimated black hole (BH) masses (see Fig. 1 in Wu et al. 2015). Some fainter candidates have been proposed, based on X-ray observations (Fiore et al. 2012; Giallongo \& et al. 2015), but they have not been confirmed yet (Weigel et al. 2015). The MBHs powering these quasars have masses in excess of $10^{8} \mathrm{M}_{\odot}$, reaching $10^{10} \mathrm{M}_{\odot}$. They are as massive as the largest MBHs today, but were in place when the Universe was less than one billion years old. The properties of quasars at $z>6$ require MBHs to form early on and grow rapidly. An initial $\mathrm{MBH}$ with mass $200 \mathrm{M}_{\odot}$ must grow continuously at the Eddington rate to reach $10^{9} \mathrm{M}_{\odot}$ by $z=7$. A seed $\mathrm{MBH}$ with mass $10^{5} \mathrm{M}_{\odot}$ needs to accrete "only" at half of the Eddington rate for the entire time, or at the Eddington rate for a half of their life. In contrast to such monsters, the MBH population extends down to small masses, though this range is hard to probe. The record for the smallest MBH currently belongs to the dwarf galaxy RGG 118, which is thought to contain a black hole weighing only $\sim 50,000 \mathrm{M}_{\odot}$ (Baldassare et al. 2015). There are also galaxies bereft of MBHs (e.g., M33, NGC 205, Gebhardt et al. 2001; Valluri et al. 2005). Therefore, models of $\mathrm{MBH}$ formation, feeding and feedback must explain both the advent of the first luminous quasars, why some galaxies host a MBH, and some others do not, and how different MBHs are fed, while providing at the same time the AGN feedback required to suppress star formation in massive galaxies. In this contribution we briefly review 


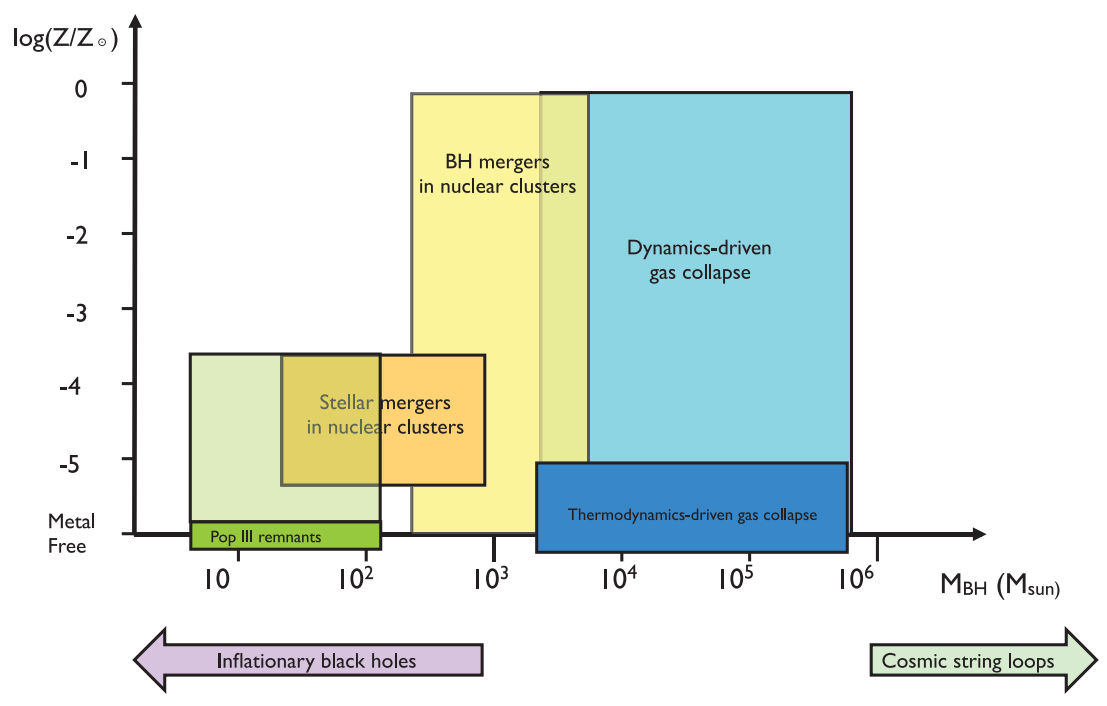

Figure 1. Cartoon summary of $\mathrm{MBH}$ formation models.

the formation, feeding and feedback of MBHs, with an emphasis on the high-redshift Universe (see also Volonteri 2012).

\section{BH formation}

Different $\mathrm{MBH}$ formation mechanisms have been proposed in the literature (see Fig. 1), starting from Rees (1984). Some proposed mechanisms, such as primordial (inflationary) MBHs (Khlopov et al. 2005) and cosmic string loops (Bramberger et al. 2015) are not directly linked to galaxy formation, and I refer the reader to the original papers for details. Recent reviews on the other processes include Volonteri (2010); Haiman (2013).

We will discuss here only one parameter relevant to $\mathrm{MBH}$ formation: the metallicity of the gas or stars that go into $\mathrm{MBH}$ formation. The crucial reason is related to stellar evolution, and specifically mass losses through winds. Yungelson et al. (2008) study the fate of solar composition stars in the mass range $60-1000 \mathrm{M}_{\odot}$. They find that they shed most of their mass via winds and are expected to end their lives as MBHs less massive than $\sim 150 \mathrm{M}_{\odot}$. At low metallicity, instead, mass loss due to winds is much more reduced, thus increasing the mass of the final remnant (Spera et al. 2015). MBH formation mechanisms that require an intermediate phase of (super)massive star, therefore, need to occur in metal poor conditions, unless the star can collapse directly through general relativistic instability (Fowler 1966; Baumgarte \& Shapiro 1999; Shibata \& Shapiro 2002). Such metallicity requirements do not apply to models that invoke stellar mass $\mathrm{MBH}$ mergers (Davies et al. 2011; Miller \& Davies 2012; Lupi et al. 2014).

One important point to keep in mind is that $\mathrm{MBH}$ formation must be common enough to account for MBHs in galaxies such as the Milky Way, as well in dwarf galaxies (Greene 2012; Reines et al. 2013). The currently fashionable models of thermodynamics-driven direct collapse at zero (e.g., Latif et al. 2013; Regan et al. 2014) or very low metallicity (Latif et al. 2015) fostered by a substantial Lyman-Werner flux seem to require very stringent conditions, leading to a very low expected number density (Habouzit et al. 2015, and references therein, but see Agarwal et al. 2015). While this mechanism, predicting very high seed masses, around $10^{5} \mathrm{M}_{\odot}$ (Lodato \& Natarajan 2007; Ferrara et al. 2014), is favoured by timescale arguments for growing high-z quasars, it seems unable to seed 

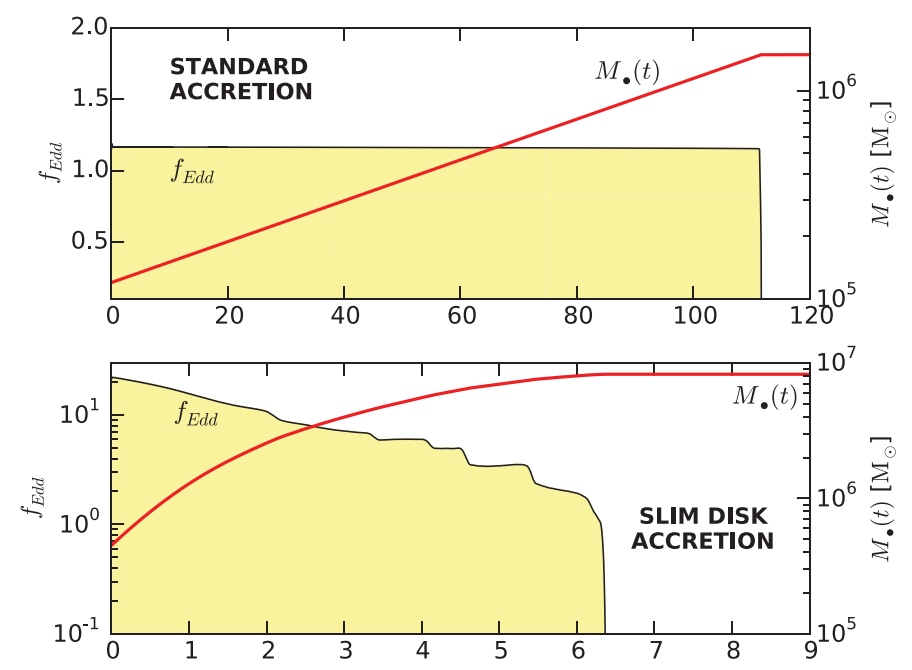

Figure 2. Time evolution of the Eddington ratio $f_{\mathrm{Edd}}$ and of the black hole mass $M_{\bullet}$, in the standard case, where $L=f_{\mathrm{Edd}} L_{\mathrm{Edd}}$ (top) and the slim disc case, where $L \sim \ln \left(f_{\mathrm{Edd}}\right) L_{\mathrm{Edd}}$ (bottom), so that luminosity remains sub-Eddington, while accretion is super-critical.

run-of-the-mill galaxies. One or more MBH formation mechanisms can occur in the Universe, though, they are not necessarily mutually exclusive (e.g., Volonteri \& Begelman 2010; Devecchi et al. 2012).

\section{BH feeding}

The very bright quasars with $z>6$ require to be fed at the Eddington level for their whole lifetime. Are such long-lived accretion events possible? Li et al. (2007), using series of successive mergers extracted from a cosmological run, suggested that mergerdriven accretion does the trick. Di Matteo et al. (2012) propose instead direct accretion from the cosmic cold flows. They use direct cosmological simulations, but with relatively low resolution $(\sim 1 \mathrm{kpc})$. At the other extreme, Bournaud et al. (2011), use very high resolution (1 pc) but isolated disc galaxy simulations to suggest that accretion is driven by disc instabilities in high-z galaxies. Finally, Dubois et al. (2012) use cosmological zooms, with a maximum resolution of $\sim 10 \mathrm{pc}$ to find that direct accretion of cosmic gas dominates early on, disc feeding takes over at later times, and galaxy mergers become important even later on.

In all these studies, the simulation provides information on the gas inflowing from the galaxy. The accretion rate on the $\mathrm{MBH}$ is calculated from this inflow rate, with an explicit cap at the Eddington luminosity. In a real galaxy, how does it know that it has to feed the $\mathrm{MBH}$ exactly at the rate resulting in the Eddington luminosity? It is important to stress that super-critical accretion does not necessarily imply highly super-Eddington luminosity. At high accretion rates the thermodynamics and geometry of the accretion disc changes (e.g., slim discs, Abramowicz et al. 1988). The material is so dense and optically thick that photons are advected inward with the gas, rather than diffuse out. Radiation is trapped, and luminosity highly suppressed (Begelman 1979): $L \propto \ln \dot{M}$ instead of $L \propto \dot{M}$. Short periods of super-critical accretion are sufficient to ease the constraints on the growth of billion solar masses MBHs at $z>6$ (e.g., Volonteri \& Rees 2005; Volonteri et al. 2015). As proof of concept, Pacucci et al. (2015) perform 1D simulation including radiation transfer of a high-z halo feeding a seed $\mathrm{MBH}$. The radiation-related 
quantities are integrated over frequencies with matter and radiation coupled via Thomson (electron) scattering and bound-free interactions. The accretion rate is calculated at the innermost cell $(0.002 \mathrm{pc})$ and the accretion and radiative efficiencies are implemented in two ways. In one series of runs, the "standard" case luminosity is calculated with a fixed matter-energy conversion factor $(\epsilon=0.1)$, and $L=f_{\mathrm{Edd}} L_{\mathrm{Edd}}$. In the "slim disc" case, only a fraction of the emitted luminosity escapes to infinity, and the disc becomes radiatively inefficient, with $\epsilon \sim 0.04$ (Mineshige et al. 2000) and $L \sim \ln \left(f_{\mathrm{Edd}}\right) L_{\mathrm{Edd}}$. The effective accretion on the MBH is modulated by gas inflows (feeding the MBH) and radiation pressure (sweeping back the gas). The evolution of $\mathrm{MBH}$ masses and the Eddington rate are shown in Fig. 2. While in standard case the $\mathrm{MBH}$ accretes $\sim 5 \%-15 \%$ of the available gas in $\sim 100 \mathrm{Myr}$, with outflows modulating the gas inflows, in the slim disc case the $\mathrm{MBH}$ accretes $>80 \%$ of the available gas in $\sim 10 \mathrm{Myr}$, with outflows playing a negligible role.

\section{BH feedback}

Feedback from MBHs is an important ingredient to understanding the relationship between galaxy and black hole growth (Silk \& Rees 1998). Winds from active MBHs find the easiest path through low-density gas, avoiding the dense parts of the galaxy disc (Gabor \& Bournaud 2014; Roos et al. 2015). Cosmological simulations show that, rather than tearing apart the galaxy, the AGN create large-scale polar outflows that eventually destroy the cold circumgalactic medium, leaving a hot halo and strangling the host galaxy's supply of cold gas (Dubois et al. 2013; Costa et al. 2014; Tremmel et al. 2015 , in prep.). This is supported by observations, as star forming galaxies are common hosts of bright AGN (Rosario et al. 2013; Mullaney et al. 2012). AGN feedback is able to explain the inefficient star formation observed in massive galaxies, causing the turn-over in stellar mass-halo mass relationships (e.g., Croton et al. 2006; Tremmel et al. 2015, in prep.).

MBHs also affect their own gas supply through feedback, possibly stunting their growth at early times (Alvarez et al. 2009; Milosavljević et al. 2009; Johnson et al. 2011; Park \& Ricotti 2012; Aykutalp et al. 2014) and eventually reaching a state of self-regulation at later times. The onset and nature of this self-regulation phase is, at least in part, governed by SN feedback and nearby gas dynamics (Dubois et al. 2015; Habouzit et al. 2015, in prep.; Tremmel et al. 2015, in prep.).

AGN can also have positive feedback on their surroundings (e.g., Zinn et al. 2013; Cresci et al. 2015), triggering star formation, for instance because of shock pressureenhanced star formation (Wagner et al. 2013; Silk 2013; Bieri et al. 2015; Gaibler et al. 2012).

An important caveat is that the implementation of AGN feedback is still very heuristic. Simulations including radiative transfer (Bieri et al. 2015, in prep.) and realistic jets (Cielo et al. 2015, in prep.), as well as all the physics of galaxy formation, will be paramount to have an accurate understanding of how feedback affects the MBH itself and the galaxy.

\section{BHs and galaxies}

The constraints on the relationship between $\mathrm{MBH}$ masses and galaxies properties at $z \simeq 6$ are few, and seem to provide conflicting results. (i) There seems to be little correlation between $\mathrm{MBH}$ mass and host properties, or at least a much larger scatter (Wang et al. 2010; Willott et al. 2015), (ii) typically MBHs are 'over-massive' at fixed 

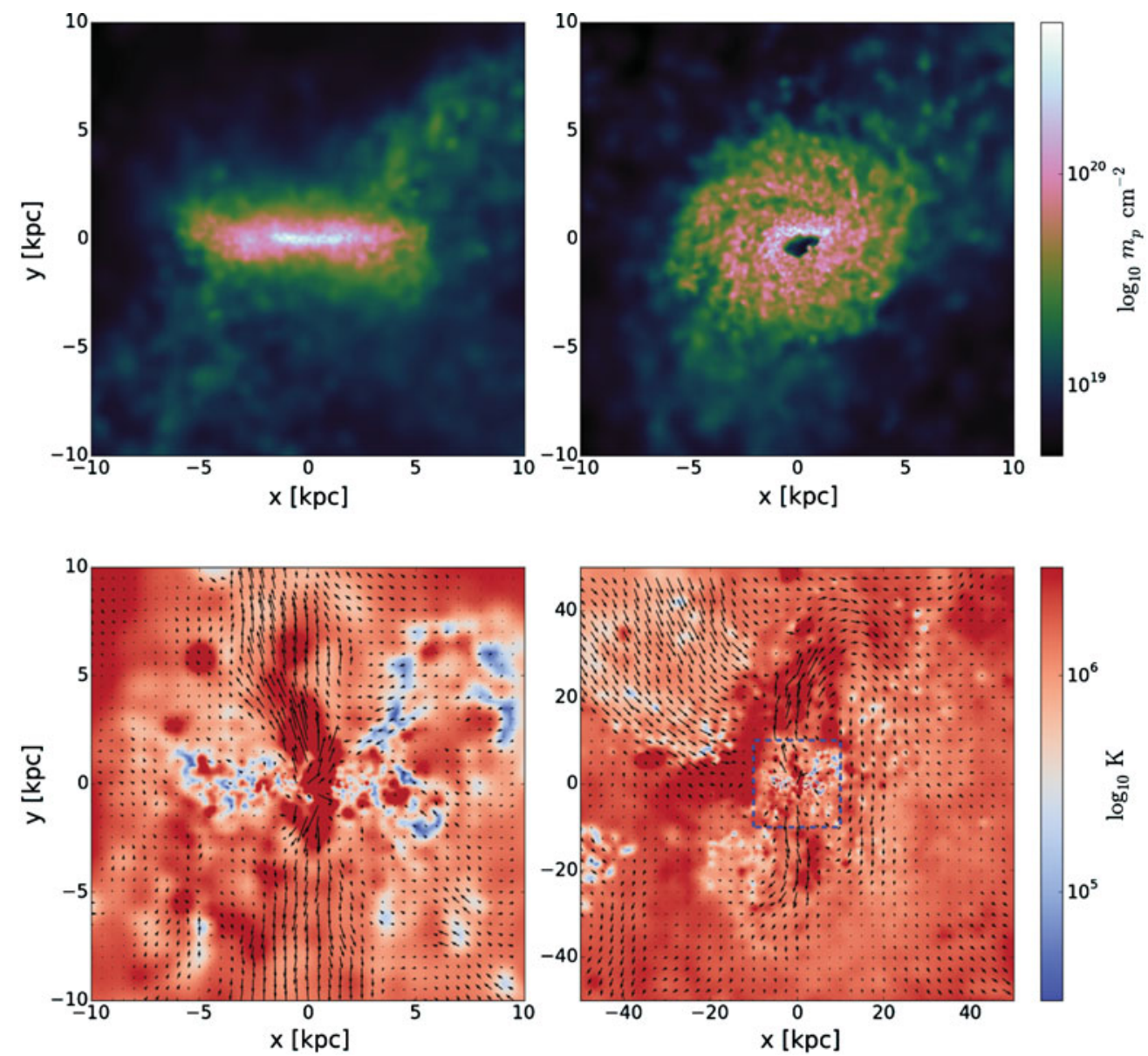

Figure 3. AGN feedback does not destroy galaxies. They drive large-scale outflows that regulate and eventually stop the flow of cold gas onto their host galaxy.

galaxy mass/velocity dispersion compared to their $z=0$ counterparts (e.g., Walter et al. 2004; at lower redshift see also McLure \& Dunlop 2004; Shields et al. 2006; Decarli et al. 2010; Merloni 2010), but (iii) analysis of the MBH mass/luminosity function and clustering suggests that either many massive galaxies do not have MBHs, or these MBHs are less massive than expected (Willott et al. 2010; Weigel et al. 2015). As a result of point (ii), most authors propose that there is positive evolution in the MBH massgalaxy relationships, and quantify it as a change in normalization, in the sense that at fixed galaxy properties (e.g. velocity dispersion, stellar mass), MBHs at high redshift are more massive than today. However, this is inconsistent with point (iii) above. Volonteri \& Stark (2011) propose that if the evolution of the correlations is in slope rather than normalization, with a tilt such that $\mathrm{MBHs}$ in small galaxies are under-massive and $\mathrm{MBHs}$ in large galaxies are over-massive, then one can reconcile the observational results (i)-(ii) and (iii) above.

While observations are still limited, cosmological simulations seem indeed to find that MBHs in small galaxies are unable to grow and remain "stuck" at low mass, as shown in Fig. 4, while MBHs in the most massive galaxies can grow beyond the correlations between MBH mass and host properties found at $z=0$ (Sijacki et al. 2015; DeGraf et al. 2015; Habouzit et al. 2015, in prep.). Indeed low-mass galaxies are a fragile environment, 


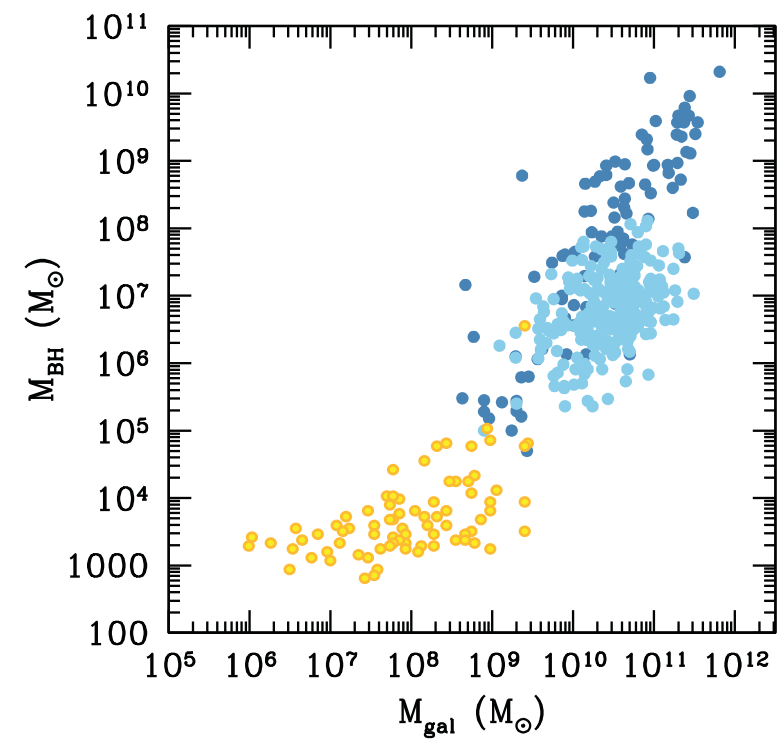

Figure 4. BH mass versus galaxy stellar mass. Dark and light blue points are quiescent MBHs and low-luminosity AGN at $z=0$ from Reines \& Volonteri (2015). Yellow points are MBHs in a cosmological simulation (using the adaptive mesh refinement code RAMSES, with a maximum resolution of $80 \mathrm{pc}$, Habouzit et al. in prep). MBHs in small galaxies are unable to grow because of the interplay between SN and AGN feedback, very effective in small galaxies' potential wells. Once a galaxy has become sufficiently massive to contrast outflows, its MBH can thrive.

and supernova feedback is sufficient to energize the gas and suppress $\mathrm{MBH}$ accretion (Dubois et al. 2015). MBHs are also easily perturbed from the center of small galaxies and experience long orbital decay timescales, making it more difficult for them to grow efficiently (Tremmel et al. 2015).

\section{Conclusions}

High redshift MBHs and quasars represent both a theoretical and observational challenge. The current sample is limited to the brightest sources, powered by extremely massive MBHs. One should appreciate that theory and observations have the same limitation: either one has a large, shallow survey (large, low-resolution simulation), or a narrow, deep survey (small, high-resolution simulation). The number density of these quasars is so low, $\sim$ one per $\mathrm{Gpc}^{3}$, that comparable volumes must be simulated in order to catch a glimpse of their evolution. Such large volumes are limited in terms of physical resolution, but we are currently reaching a good balance between size and spatial information (Feng et al. 2015). Several telescopes in the near future promise to greatly increase the sensitivity, thus being able to detect lower luminosity AGN, hopefully powered by lower mass MBHs (Pacucci et al. 2015). JWST, ATHENA and SKA are all geared towards finding and studying the high redshift Universe, and the $\mathrm{MBHs}$ and galaxies that inhabit it. Meanwhile, $A L M A$ can provide us with unprecedented information on the dynamics and kinematics of molecular gas in the galaxies hosting quasars and AGN. On the theoretical side, large volumes at high-resolution are becoming a reality, and we can now shift the attention from reproducing statistical results, to try and understand the physics of $\mathrm{MBH}$ formation, feeding and feedback. 


\section{Acknowledgments}

MV acknowledges funding from the European Research Council under the European Community's Seventh Framework Programme (FP7/2007-2013 Grant Agreement no. 614199, project "BLACK").

\section{References}

Abramowicz M. A., Czerny B., Lasota J. P., \& Szuszkiewicz E., 1988, ApJ, 332, 646 Agarwal B., Smith B., Glover S., Natarajan P., \& Khochfar S., 2015, ArXiv e-prints Alvarez M. A., Wise J. H., \& Abel T., 2009, ApJ, 701, L133

Aykutalp A., Wise J. H., Spaans M., \& Meijerink R., 2014, ApJ, 797, 139

Baldassare V. F., Reines A. E., Gallo E., \& Greene J. E., 2015, ApJ, 809, L14

Baumgarte T. W. \& Shapiro S. L., 1999, ApJ, 526, 941

Begelman M. C., 1979, MNRAS, 187, 237

Bieri R., Dubois Y., Silk J., \& Mamon G., Gaibler V., 2015, ArXiv e-prints

Bieri R., et al. 2015, in prep.

Bournaud F., Dekel A., Teyssier R., et al. 2011, ApJ, 741, L33

Bramberger S. F., Brandenberger R. H., Jreidini P., \& Quintin J., 2015, JCAP, 6, 7

Cielo R., et al. 2015, in prep.

Costa T., Sijacki D., \& Haehnelt M. G., 2014, MNRAS, 444, 2355

Cresci G., Marconi A., Zibetti S., et al. 2015, AछA, 582, A63

Croton D. J., Springel V., White S. D. M., et al. 2006, MNRAS, 365, 11

Davies M. B., Miller M. C., \& Bellovary J. M., 2011, ApJ, 740, L42

Decarli R., Falomo R., Treves A., et al. 2010, MNRAS, 402, 2453

DeGraf C., Di Matteo T., Treu T., Feng Y., Woo J.-H., \& Park D., 2015, MNRAS, 454, 913

Devecchi B., Volonteri M., Rossi E. M., Colpi M., \& Portegies Zwart S., 2012, MNRAS, 421, 1465

Di Matteo T., Khandai N., DeGraf C., Feng Y., et al. 2012, ApJ, 745, L29

Dubois Y., Pichon C., Devriendt J., et al. 2013, MNRAS, 428, 2885

Dubois Y., Pichon C., Haehnelt M., et al. 2012, MNRAS, 423, 3616

Dubois Y., Volonteri M., Silk J., Devriendt J., Slyz A., \& Teyssier R., 2015, MNRAS, 452, 1502

Feng Y., Di-Matteo T., Croft R. A., Bird S., Battaglia N., \& Wilkins S., 2015, ArXiv e-prints

Ferrara A., Salvadori S., Yue B., \& Schleicher D., 2014, MNRAS, 443, 2410

Fiore F., Puccetti S., Grazian A., et al. 2012, A\&A, 537, A16

Fowler W. A., 1966, ApJ, 144, 180

Gabor J. M. \& Bournaud F., 2014, MNRAS, 441, 1615

Gaibler V., Khochfar S., Krause M., \& Silk J., 2012, MNRAS, 425, 438

Gebhardt K., Lauer T. R., Kormendy J., et al. 2001, AJ, 122, 2469

Giallongo E., et al. 2015, A\& $A, 578, \mathrm{~A} 83$

Greene J. E., 2012, Nature Communications, 3, 1304

Habouzit M. \& Volonteri M., et al. 2015, in prep.

Habouzit M., Volonteri M., Latif M., et al. 2015, ArXiv e-prints

Haiman, Z. 2013, Astrophysics and Space Science Library, 396, 293

Johnson J. L., Khochfar S., Greif T. H., \& Durier F., 2011, MNRAS, 410, 919

Khlopov M. Y., Rubin S. G., \& Sakharov A. S., 2005, Astroparticle Physics, 23, 265

Latif M. A., Omukai K., Habouzit M., Schleicher D. R. G., \& Volonteri M., 2015, ArXiv e-prints

Latif M. A., Schleicher D. R. G., Schmidt W., \& Niemeyer J., 2013, MNRAS, 433, 1607

Li Y., Hernquist L., Robertson B., et al. 2007, ApJ, 665, 187

Lodato G. \& Natarajan P., 2007, MNRAS, 377, L64

Lupi A., Colpi M., Devecchi B., Galanti G., \& Volonteri M., 2014, MNRAS, 442, 3616

McLure R. J. \& Dunlop J. S., 2004, MNRAS, 352, 1390

Merloni A. e. a., 2010, ApJ, 708, 137

Miller M. C. \& Davies M. B., 2012, ApJ, 755, 81 
Milosavljević M., Couch S. M., \& Bromm V., 2009, ApJL, 696, L146

Mineshige S., Kawaguchi T., Takeuchi M., \& Hayashida K., 2000, PASJ, 52, 499

Mortlock D. J., Warren S. J., Venemans B. P., et al., 2011, Nature, 474, 616

Mullaney J. R., Daddi E., Béthermin M., et al. 2012, ApJ, 753, L30

Pacucci F., Ferrara A., Volonteri M., \& Dubus G., 2015, ArXiv e-prints

Pacucci F., Volonteri M., \& Ferrara A., 2015, MNRAS, 452, 1922

Park K. \& Ricotti M., 2012, ApJ, 747, 9

Rees M. J., 1984, ARA\&A, 22, 471

Regan J. A., Johansson P. H., \& Wise J. H., 2014, ApJ, 795, 137

Reines A. E., Greene J. E., \& Geha M., 2013, ApJ, 775, 116

Reines A. E. \& Volonteri M., 2015, ArXiv e-prints

Roos O., Juneau S., Bournaud F., \& Gabor J. M., 2015, ApJ, 800, 19

Rosario D. J., Trakhtenbrot B., Lutz D., et al. 2013, A\&A A, 560, A72

Sądowski, A., Narayan, R., McKinney, J. C., \& Tchekhovskoy, A. 2014, MNRAS, 439, 503

Shibata M. \& Shapiro S. L., 2002, ApJ, 572, L39

Shields G. A., Menezes K. L., Massart C. A., \& Vanden Bout P., 2006, ApJ, 641, 683

Sijacki D., Vogelsberger M., Genel S., et al., 2015, MNRAS, 452, 575

Silk, J. \& Rees, M. J. 1998, A\&SA, 331, L1

Silk J., 2013, ApJ, 772, 112

Spera M., Mapelli M., \& Bressan A., 2015, MNRAS, 451, 4086

Tremmel M., Governato F., et al. 2015, in prep.

Tremmel M., Governato F., Volonteri M., \& Quinn T. R., 2015, MNRAS, 451, 1868

Valluri M., Ferrarese L., Merritt D., \& Joseph C. L., 2005, ApJ, 628, 137

Volonteri M., 2010, A\&AR, 18, 279

Volonteri M., 2012, Science, 337, 544

Volonteri M. \& Begelman M. C., 2010, MNRAS, 409, 1022

Volonteri M. \& Rees M. J., 2005, ApJ, 633, 624

Volonteri M., Silk J., \& Dubus G., 2015, ApJ, 804, 148

Volonteri M. \& Stark D. P., 2011, MNRAS, 417, 2085

Wagner A. Y., Umemura M., \& Bicknell G. V., 2013, ApJ, 763, L18

Wang R., Carilli C. L., Neri R., et al., 2010, ApJ, 714, 699

Weigel A. K., Schawinski K., Treister E., et al. 2015, MNRAS, 448, 3167

Willott C. J., Albert L., Arzoumanian D., et al. 2010, AJ, 140, 546

Willott C. J., Bergeron J., \& Omont A., 2015, ApJ, 801, 123

Wu X.-B., Wang F., Fan X., et al. 2015, Nature, 518, 512

Yungelson L. R., van den Heuvel E. P. J., Vink J. S., et al., 2008, A\&AA, 477, 223

Zinn P.-C., Middelberg E., Norris R. P., \& Dettmar R.-J., 2013, ApJ, 774, 66 